\title{
Experimentelle Untersuchungen über das Schicksal der Tuberkelbazillen im Blutstrom, insbesondere über ihre Ausscheidungswege.
}

Mitteilung. I: Über die Verbreitung von den in die Ohrvene injizierten Tuberkelbazillen in der Lunge der Kaninchen, insbescndere ibr Auftreten im Alveolar- und Bronchiallursen.

Von

\section{Takesi Abe. \\ (阿 部 武)}

(Aus der Medizinischen Klinik und dem Kosankinbyo

Kenkyusho der Kaiserlichen Tohoku-Universitat

zu Sendai, Leiter: Prof. Dr. T. Kumaga i und Prof. Dr. T. Ebina)

(Received for publication, April 4, 1945)

\section{Einleitung.}

Das Schicksal der Tuberkelbazillen im Blutstrom wurde von vielen Autoren, Baumgarte ${ }^{1)}$ an der Spitze, experimentell untersucht und diesbezügliche Angaben sind zu zahlreich, um einzeln aufgeführt werden zu konnen. Die meisten Autoren haben jedoch hauptsächlich die Verbreitung der Tuberkelbazillen oder ihre Affinität mit verschiedenen Organen untersucht, aber über die Ausscheidung der Bazillen liegen nur einzelne Angaben vor. Namentlich über die Ausscheidung durch die Luftwege gibt es zwar die Angabe von Goyal, ${ }^{2)}$ der Tuberkelbazillen aus dem Trachealsekret züchtete, allein unseres Wissens noch keine, wo man die im Alveolar- oder Bronchiallumen frei liegenden Tuberkelbazillen histologisch nachgewiesen hätte.

In vorliegender Arbeit wollen wir zuerst bei Kaninchen mit der Bazillämie, die durch intraven öse Injektion der Tuberkelbazillen künstlich verursacht wird, die Verbreitung von Tuberkelbazillen in der Lunge und ihr Schicksal, besonders ihr Auftreten im Alveolar- und Bronchiallumen, ihre Ausscheidung durch die Luftwege untersuchen.

2. Versuchsmaterial und -methode.

Als Versuchstiere wurden 37 gesunde Kaninchen mit negativer Tuberkulinreaktion benutzt. Hoch virulente Tuberkelbazillen vom Typus humanus (,, Sato "-Stamm) wurden so homogen emulsiert, dass 
in $1 \mathrm{ccm}$ Emulsion 1 oder $10 \mathrm{mg}$ Bazillen enthalten waren. $1 \mathrm{ccm}$ von diesen Emulsionen wurde Kaninchen in die Ohrvene eingespritzt, und die Versuchstiere wurden danach in bestimmten Zeiträumen durch die Luftembolie getötet. Die exstirpierten Organe wurden zuerst mit 10\% Formollösung fixiert, dann wurden wie gewöhnlich Paraffin-Schnitte hergestellt. Sie wurden nach der Haematoxylin-Eosin-Doppelfärbung, der Weig er tschen Färbung elastischer Fasern, der va n Gi es o nschen

\section{TABELLE. I.}

Tab. 1. Lungenbefund der Kaninchen mit der Bazillämie.

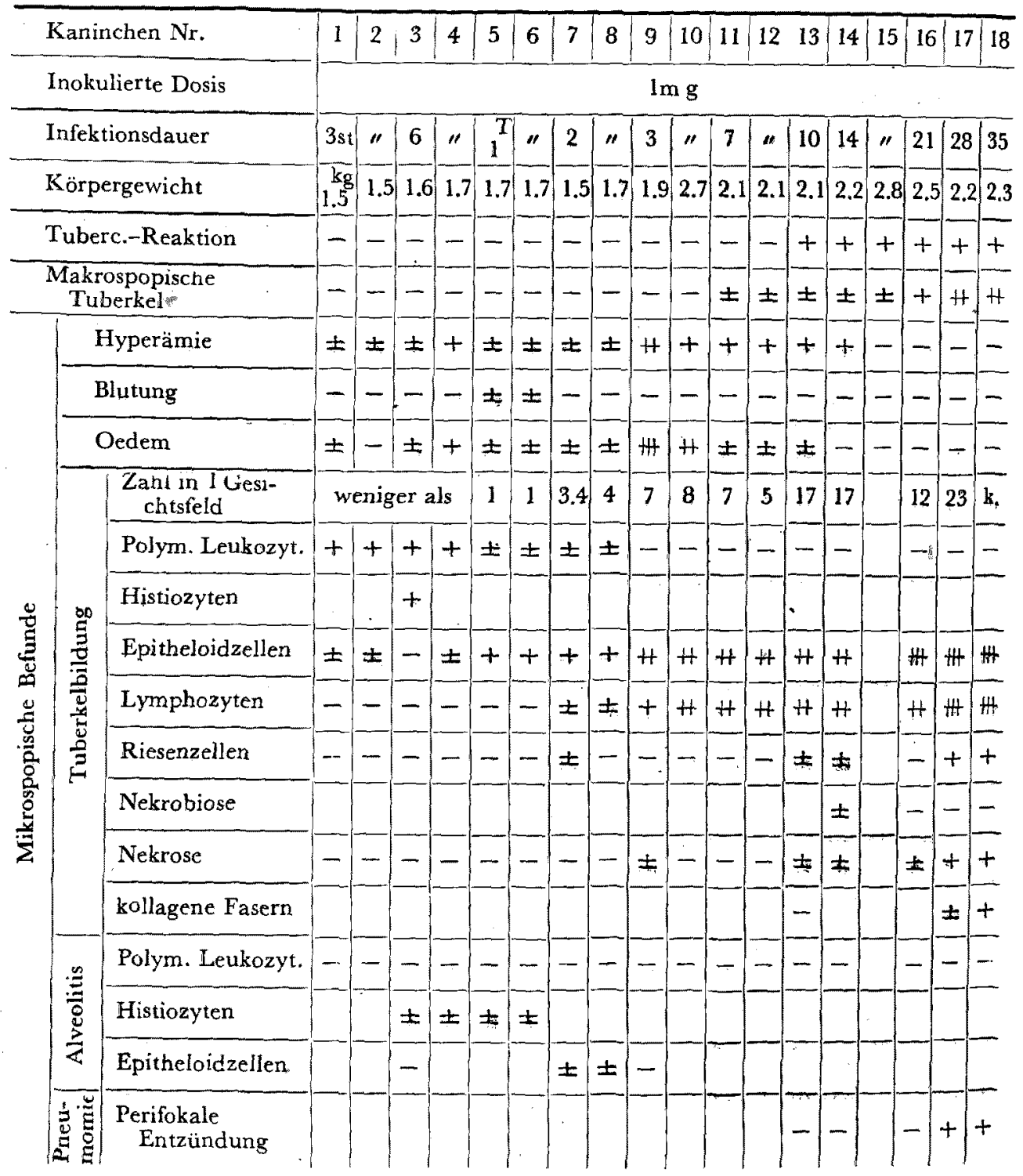


TABELLE I.

(Fortgesetzt)

\begin{tabular}{|c|c|c|c|c|c|c|c|c|c|c|c|c|c|c|c|c|c|c|c|c|}
\hline \multirow{2}{*}{\multicolumn{3}{|c|}{$\frac{\text { Kaninchen Nr. }}{\text { Inokulierte Dosis }}$}} & 19 & 20 & 21 & 22 & 23 & $24 \mid 2$ & 252 & 26 & $27 \mid 2$ & 2829 & 930 & 31 & 32 & 33 & 34 & 35 & 36 & 37 \\
\hline & & & \multicolumn{18}{|c|}{$10 \dot{\mathrm{mg}}$} \\
\hline \multicolumn{3}{|c|}{ Infektionsdauer } & $1 s t$ & 3 & 6 & $24 / 2$ & 2 & 3 & 7 & 8 & 10 & 1113 & 314 & 16 & 21 & 28 & 34 & 41 & 41 & 44 \\
\hline \multicolumn{3}{|c|}{ Körpergewicht } & 1.5 & 1.7 & 1.9 & 1.4 & 2.0 & 1.9 & 2.1 & 1.4 & 2.01 & 1.52 & 31. & 42.0 & 2.1 & 1.5 & 1.4 & 2.5 & 2.2 & 1.5 \\
\hline \multicolumn{3}{|c|}{ Tuberc.-Reaktion } & - & - & - & - & - & - & + & + & +1 & ++ & ++ & + & + & + & + & + & + & + \\
\hline \multicolumn{3}{|c|}{$\begin{array}{c}\text { Makrospopische } \\
\text { Tuberkel }\end{array}$} & - & - & - & - & - & - & + & + & +1 & ++ & + & + & + & $H$ & $H$ & + & + & $H$ \\
\hline \multicolumn{3}{|c|}{ Hyperämie } & $H$ & + & + & - & - & in & + & + & $+=$ & \pm \pm & $=1+$ & $\neq$ & - & - & - & + & + & \pm \\
\hline \multicolumn{3}{|c|}{ Blutung } & \pm & \pm & - & - & - & +1 & \pm & - & $-=$ & \pm- & $-1-$ & - & - & - & - & - & - & - \\
\hline \multicolumn{3}{|c|}{ Oeden } & $H$ & H & + & - & $H$ & $H$ & $H$ & $H$ & + & $-H$ & $+1-$ & $-1-$ & -1 & - & - & H & + & \pm \\
\hline \multirow{9}{*}{\multicolumn{2}{|c|}{ 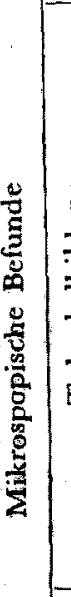 }} & $\begin{array}{l}\text { Zahl in } 1 \text { Gesi- } \\
\text { chtsfeld }\end{array}$ & \begin{tabular}{|l} 
wen \\
als
\end{tabular} & igel & 4.4 & $H$ & 2,7 & 14 & 5.5 & \pm & 412 & 2625 & $5 \mid 23$ & $\begin{array}{l}3 \\
3\end{array}$ & \multicolumn{4}{|c|}{ Konfluiert } & & \\
\hline & \multirow{5}{*}{ 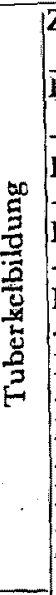 } & $\begin{array}{l}\text { Polym. Leuko- } \\
\text { zyt. }\end{array}$ & + & + & + & 1.5 & +1 & $H$ & \pm & $t$ & $-=$ & \pm- & $-1-$ & $1-$ & - & - & - & - & - & - \\
\hline & & Histiozyten & + & - & - & $H$ & - & - & - & - & - - & - & - & - & - & - & - & - & - & - \\
\hline & & Epitheloidzellen & $1-1$ & + & + & - & $H$ & 4 & $H$ & $H$ & $H+$ & $H: H$ & $H$ & $H$ & H & 4 & \# & H & m & \# \\
\hline & & Lymphozyten & - & - & - & + & \pm & + & $H$ & $H$ & $H+$ & $H: H$ & $+H$ & + & + & H & H & $H$ & 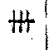 & H \\
\hline & & Riesenzellen & - & - & - & - & \pm & \pm & - & \pm & - & - & - & \pm & - & - & - & - & - & + \\
\hline & & Nekro & -1 & $1-$ & - & - & - & - & - & - & - & - \pm & $=+$ & + & + & + & + & & & \\
\hline & & Nekrose & - & - & - & - & - & - & - & \pm & - & - & \pm & $=+$ & + & + & H & $H$ & $H$ & H \\
\hline & & $\begin{array}{c}\text { Konlagene } \\
\text { Fasern }\end{array}$ & - & - & - & - & - & - & - & - & $\pm=$ & \pm & + & + & - & + & \pm & +1 & + & \pm \\
\hline & $:$ & $\begin{array}{l}\text { Polym. Leuko- } \\
\text { zyt. }\end{array}$ & - & - & -1 & - & -1 & - & - & - & & - & - & -1 & - & - & - & $\ldots$ & -1 & - \\
\hline & "0 & Histiozyten & - & - & - & - & - & + & - & & & & & & & & & & & \\
\hline & 4 & Epitheloidzellen & - & - & - & - & - & + & - & + & - & - & - & +4 & $H$ & $H$ & $H$ & & & \\
\hline & 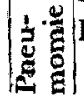 & $\begin{array}{l}\text { Perifokale } \\
\text { Entzünd }\end{array}$ & $1-$ & & -1 & - & +1 & -1 & -1 & - & - & $-i$ & - & $-1+$ & + & +1 & $H$ & H & $H$ & 7 \\
\hline
\end{tabular}

Anmerkungen: \pm ein wenig, +mittelmässig, Hziemlich, Hhochgradig.

Färbung und der von Katura modifizierten TuberkelbazillenFärbung im Gewebe von $\mathrm{Wada}^{3)}$ gefärbt und mikroskopiert. Bei der Sektion von den Fällen, denen $10 \mathrm{mg}$ Bazillen eingesprizt wurde, schnitt man die Trachea und brannte die Schnittflächen mit einer glühenden Öse aus, damit Blut nicht überströmte. Dann führte man eine mit $2 \%$ $\mathrm{NaOH}$ angeklebte sterilisierte Öse in die Trachea ein, um das Innere 
der letzteren mit $\mathrm{NaOH}$ nass' genug zu machen. Nachdem das Sekret geschmolzen war, entnahm man es mit einer Öse. Nach Zusatz von ungefähr $10 \mathrm{ccm} 2 \% \mathrm{NaOH}$ und ca $1 \mathrm{ccm} 2.5 \% \mathrm{ZnSO}_{4}$ wurde das Versuchsmaterial 30 Minuten lang stehen gelassen. Dann wurde es 10 Minuten lang mit 3000 Drehungen per Minute zentrifugiert. Dem Niederschlag wurde 2 oder $3 \mathrm{ccm} 4 \% \mathrm{H}_{2} \mathrm{SO}_{4}$ tropfenweise zugesetzt. Nach 10 Minuten langem Stehenlassen wurde die ganze Menge vom Niederschlag auf den Oka-Katakura schen Nährboden ausgestrichen.

\section{Versuchsergebnisse.}

1) Hyperämie, Blutung und Oedem. Nach Ablauf von einer Stunde nach der Injektion von Tuberkelbazillen tritt die Hyperämie und teilweise Blutung auf. Sie wird nach 3 Tagen stärker und bei den Fällen, die $1 \mathrm{mg}$ Bazillen bekamen, nach einer Woche und bei denjenigen, die 10 mg Bazillen erhielten, nach 10 Tagen schwächer und nach 3-4 Wochen nicht mehr bemerkt.

Eine Stunde nach der Einspritzung von Bazillen tritt die Odemflüssigkeit ins Alveolarlumen ein. Sie nimmt nach 3-6 Stunden erheblich zu, aber nach ungefähr 10 Tagen ab und wird meist nach 2-3 Wochen fast nicht erkannt.

2) Tuberkelbildung. a) Tuberkelbildung im Interstitium.

Eine Stunde nach der Bazilleninjektion entstenhen im Alveolarseptum sehr kleine Herde, die als Anfänge der Tuberkelbildung anzusehen sind. Nach 3-6 Stunden vergrössern und vermehren sie sich, und zwar wird innerhalb 24 Stunden bei einer schwachen Vergrösserung durchschnittlich nur weniger als ein Herd in einem Gesichtsfeld, wohl aber nach 48 Stunden 2-3 und nach einer Woche ungefähr 5 Herde wahrgenommen. Nach 10 Tagen zählen sie bei den Tieren, die $1 \mathrm{mg}$ Bazillen bekamen, ungefähr 7 und bei denjenigen, denen $10 \mathrm{mg}$ Bazillen injiziert wurden, 26-41. Von diesem Zeitpunkte an nehmen sie nicht mehr erheblich zu und beginnen nach 3-4 Wochen zu konfluieren.

Die Tuberkel konstruiert sich bis zum Ablauf von 6 Stunden nach der Injektion aus polymorphkernigen Leukozyten und wenigen mononu"kleären Zellen, welche als junge Epitheloidzellen anzusehen sind, urid nach 24 Stunden hauptsächlich bald aus polymorphkernigen Leukozyten, bald Epitheloidzellen; nach 48 Stunden finden sich ferner auch eine geringe Menge Lymphozyten und selten Riesenzellen. Nach 3 Tagen werden bei der Injektion von $1 \mathrm{mg}$ Bazillen fast keine polymorphkernigen Leukozyten gefunden. Bei der von $10 \mathrm{mg}$ Bazillen aber sind nach 8-11 Tagen noch wenige Leukozyten gemischt, und erst nach 13 Tagen sind sie nicht mehr enthalten. Die Nekrose kann bei seltenen Fällen schon nach 3 Tagen, aber gewöhnlich nach 10-14 Tagen deutlich vorkommen, nachdem die 
Piknose und Nekrobiose aufgetreten sind.

b) Tubelkelbildung im Alveolarlumen. Schon 3 Stunden nach der Bazilleninjektion treten im Alveolarlumen grosse mononukleäre Zellen auf, die in Form und Grösse den grossen mononukleären Zellen im Alveolarseptum gleich und daher wahrscheinlich Histiozyten sind. Sie nehman nach 6 Stunden verhältnismässig zu und sind nach 48 Stunden noch zu finden. Nach 2-3 Tagen erscheinen hier die Epitheloidzellen und im gegenüberliegenden Alveolarseptum Infiltrationen mit polymorphkernigen Leukozyten und grossen mononukleären Zellen. Nach 8 oder 16 Tagen vermehren sich die Epitheloidzellen erheblich und bilden Tuberkeln.

3) Bildung der pneumonischen Herde. Nach der Bazilleninjektion infiltrieren schon nach Ablauf von 48 Stunden, spätestens von 13 Tagen polymorphkernige Leukozyten, grosse mononukleäre Zellen und Lymphozyten um die Tuberkeln und bilden sog. perifokale pneumonische Herde, wodurch die Tuberkeln nach 5 Wochen konfluieren, grosse Herde bilden und eine erhebliche Verkäsung herbeigeführt wird.

4) Bronchitis. Eine Stunde nach der Einspritzung beobachtet man

TABELLE II.

Verbreitung der Tuberkelbazillen in der Lunge und Resultate der Kultur aus dem Trachealsekret.

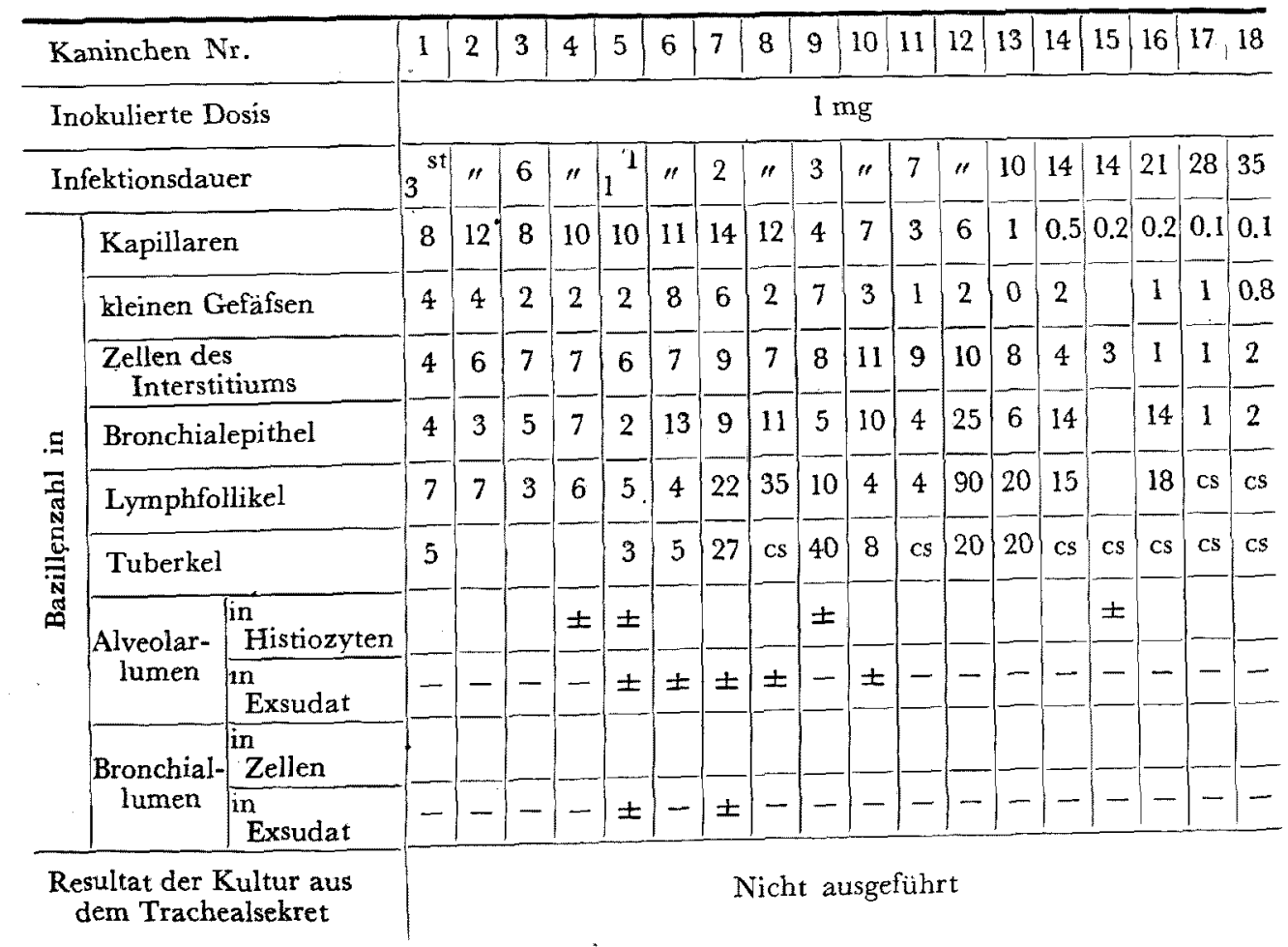


TABELLE II.

(Fortgesetzt)

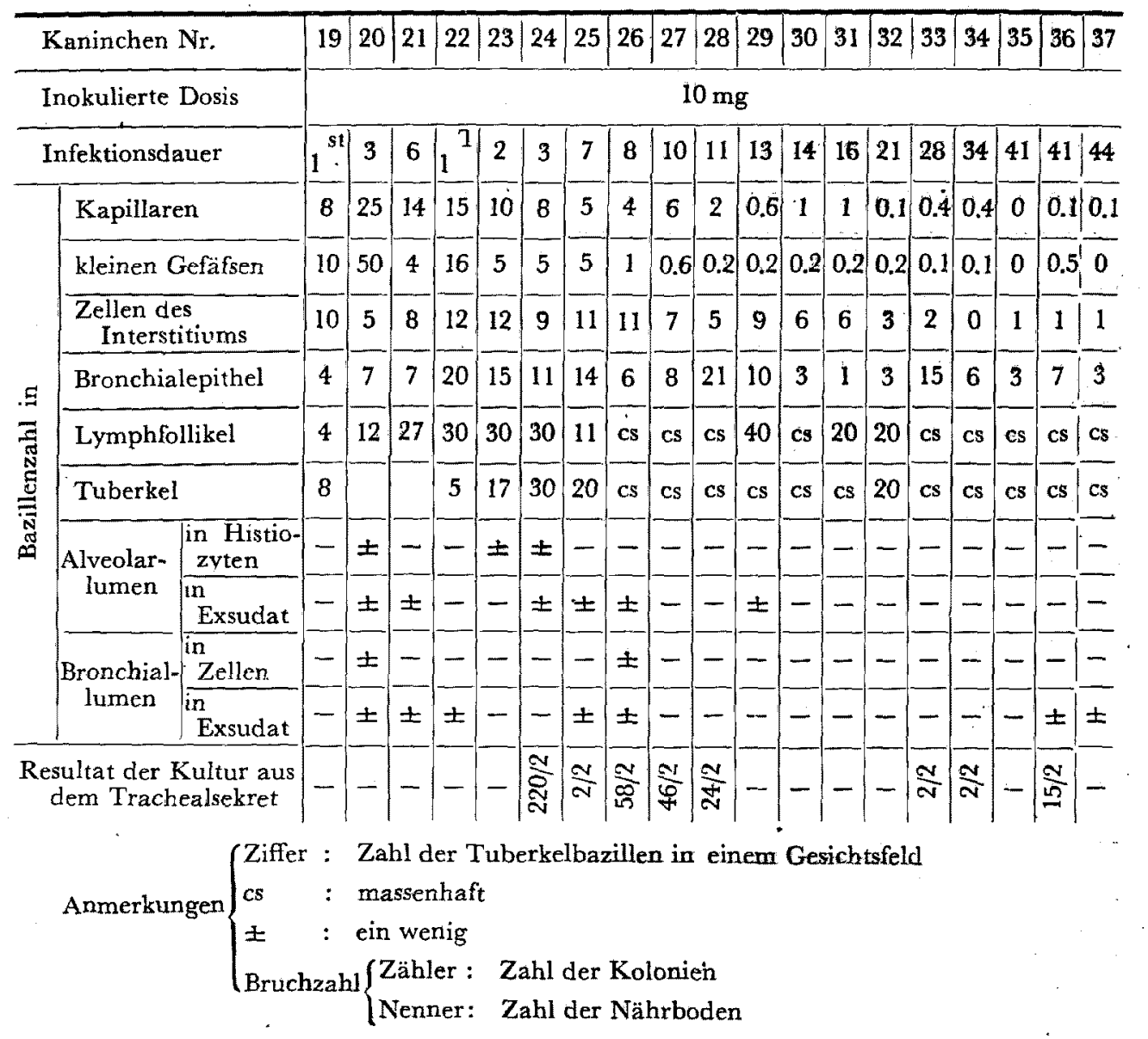

schon die Desquamation von Bronchialepithelien. Nach 3 und 24 Stunden findet sich in den Bronchien ebenfalls Exsudat, in dem nekrotische und zerfallene Zellelemente gemischt sind. Nach einer Woche wird die Bronchitis deutlich und nach 3 Wochen ziemlich stark, und nach 41 Tagen sind die Bronchien manchmal mit eitrigem Exsudat ausgefüllt.

5) Verbreitung der Tuberkelbazillen in der Lunge, insbesondere ihr Auftreten in Alveolarlumen und Bronchien.

Die Tuberkelbazillen verbreiten sich schon eine Stunde nach der Injektion in der Kapillaren der Alveolarwände, den kleinen Gefässen und Zellen des Interstitiums sowie in den Bronchialepithelzellen und den Lymphfollikeln um die Bronchien. Nach 3 Stunden werden sie im Alveolarlumen, in Exsudat oder von den grossen mononukleären Zellen gefressen, und ebenfalls auch im Bronchiallumen gefunden. 
6) Form und Schicksal der in der Lunge verbreiteten Tuberkelbazillen.

Die Tuberkelbazillen in der Kapillaren sind einzeln getrennt gelegen, manche darunter sind von polymorphkernigen Leukozyten umgeben und gefressen. Sie nehmen von 7 Tagen nach der Injektion an etwas, von 10 Tagen an erheblich und nach 3 Wochen stark ab. Die Bazillen in den Zellen des Interstitiums des Alveolarseptums sind grösstenteils granulös, besonders viele davon sind von den Histiozyten gefressen. Die Bazillen in der Bronchialepithelzellen vermehren sich nach 48 Stunden verhältnismässig und erreichen nach 11 Tagen das Maximum, um nach 2 Wochen und später abzunebmen. Die Bazillen in den Tuberkeln sind zwar im Frühstadium von den Leukozyten, aber bald danach von den grossen mononukleären Zellen umgeben und gefressen. Nach 48 Stunden nehmen sie allmählich zu und wuchern nach $7-8^{\circ}$ Tagen zahllos und büschelförmig. In den Lymphfollikeln um die Bronchien werden viele Bazillen zuerst von den Retikulumzellen granulös gefressen. Sie vermehren sich nach 48 Stunden und wuchern im allgemeinen nach 7-8 Tagen massenhaft.

Im Alveolarlumen werden nach Ablauf von 3, 6, 24, 48 und 72 Stunden freie Tuberkelbazillen erblickt, danach werden sie bei der Injektion von $1 \mathrm{mg}$ Bazillin nicht mehr erkannt, während sie bei der von 10 mg Bazillen nach 7, 8, 13 und 14 Tagen noch in geringer Menge zu finden sind und später aber werden sie nicht mehr nachgewiesen. Im Bronchiallumen befinden sie sich nach 3,6,24 und 48 Stunden. Ausserdem werden sie bei der Einspritzung von $10 \mathrm{mg}$ Bazillen nach 7-13 Tagen.gefunden und danach bis 4 Wochen nicht; aber nach 4 Wochen und später werden sie wieder aus dem Exsudat mit Zellelementen zusammen nachgewiesen.

7) Resultate der Kultur aus dem Trachealsekret. Bei den Fällen, die $10 \mathrm{mg}$ Bazillen bekamen, fällt die Kultur aus dem Trachealsekret nach 72 Stunden, 7, 8, 10 und 11 Tagen fortdauernd positiv aus und danach negativ, um aber nach 28,34 und 41 Tagen wieder positiv zu werden.

\section{Betrachtung.}

1) Weiger $\mathrm{t}^{4)}$ meinte, dass die Wirkung der Tuberkelbazillen auf das -Lungengewebe mit der primëren Gewebsschädigung beginnt und infolgedessen die Wucherung der fixen Gewebszellen herbeigeführt wird. Dagegen behauptete Baumgarten,1) dass die Tuberkelbazillen die Wucherung des Gewebes hervorrufen, ohne eine solche primäre Gewebsschädigung herbeizuführen. In der neueren Zeit stellte $\mathrm{Page}^{5}$ ) einen Dualismus auf, indem er behauptete, dass die Wucherung der fixen Gewebszellen der Lunge mit der primären Schädigung der fixen Gewebszellen gleichzeitig stattfindet. 
Wir haben Kaninchen die möglichst homogenen Emulsionen von Tuberkelbazillen vom Typus humanus in die Ohrvene injiziert und nach Ablauf von einer Stunde und später beobachtet. Dabei wurden schon im frühen Stadium die Hyperämie der Kapillaren und kleinen Gefässe der Lunge, die parenchymatöse Blutung ohne Zerstörung der Gefässwände an manchen Stellen und das erhebliche Ödem konstatiert. Wenn es auch nicht klar ist, ob es sich diese Veränderung der Zirkulation um eine Zerstörung oder eine Reaktion handelt, schliessen wir darauf, dass hier eine primäre Störung des Gewebes durch das Eindringen von Tuberkelbazillen und ihren Giftstoff liegt.

2) Orth' sagte, dass, wenn auch die 'Veränderung duch die Tuberkelbazillen im Alveolarseptum die Tuberkelbildung ist, dieselbe im Alveolarlumen eine exsudative Veränderung ist und die käsige Pneumonie hier auftritt. Baumgarten, ${ }^{1)} \mathrm{Page}^{\left.{ }^{5}\right)}$ und andere behaupteten aber, dass die beiderartige Veränderungen durch einen gleichen Prozess auftreten und die Krankheitsform nur je nach dem Virulenz der Tuberkelbazillen, der Immunität der Individuen, der eigentümlichen. Gewebskonstruktur und der Empfindlichkeit gegen die Infektion verschieden ist.

Aus unseren Versuchen ergab sich, dass die Miliartuberkel im sehr frühen Stadium im Interstitium, besonders in der Lage der Kapillaren des Alveolarseptums oder in den Wänden kleiner Gefässe und den Lymphfollikeln um die Brochien entsteht und etwas später, d.h. 48-72 Stunden nach der Injektion die Epitheloidzellen im Alveolarlumen erscheinen und dadurch die Tuberkeln bilden. Zugleich erscheinen mit dem zeitlichen Ablauf, d.h. nach 48 Stunden- 13 Tagen Zellen im Alveolarlumen nahe von Tuberkeln und bilden sich die pneumonischen Herde. Nach 5 Wochen entstehen grosse Herde durch die Konfluierung der Tuberkeln und dazu noch erhebliche Verkäsung. Bei der hämatogenen Infektion besonders im Frühstadium spielt also die produktive Veränderung die Hauptrolle und ist die exsudative Veränderung wahrscheinlich nur die perifokale Entzündung.

3) Über die Verbreitung der in den Blutstrom eingedrungenen Tuberkelbazillen in der Lunge gibt es im grossen und ganzen 4 verschiedene Ansichten. Ba umgarte $\mathrm{n}^{1)}$ meinte nämlich, dass die Tuberkelbazillen zuerst die Lymphfollikel erreichen urd dort einen Herd bilden. Nach $\mathrm{Töppich}{ }^{7)}$ werden sie von den Endothelzellen aufgenommen und nach $\mathrm{Tak}$ a m u $\mathrm{a}^{8)}$ von polymorphkernigen Leukozyten umgeben und bleiben in den Kapillaren, um hier eine Krankheitsveränderung. hervorzurufen. Miyata ${ }^{97}$ stellte fest, dass die Tuberkelbazillen nicht nur in den Blutgefässen bleiben oder die Alveolarepithelien und Lymphfollikel erreichen, sondern auch von grossen mononukleären Zellen gefressen in das Alveolarlumen gelangen. $\mathrm{Huebschmann},{ }^{10)} \mathrm{Pagel},{ }^{5)} \mathrm{Takeda}^{11)}$ und andere 
behaupteten, dass die Tuberkelbazillen theoretisch aus dem Blutstrom ins Alveoarlumen ausgeschieden werden müssen. Wenn auch Go y a ${ }^{2}$, berichtete, dass ihm der Nachweis von Tuberkelbazillen aus dem Trachealsekret im Frühstadium gelang, scheint doch noch keine Angabe vorzuliegen, in der man im Alveolarlumen frei liegende Tuberkelbazillen konstatiert habe.

In unseren Versuchen konnte man feststellen, dass die Tuberkelbazillen schon eine Stunde nach der Injektion ausser in den Kapillaren noch im Alveolarinterstitium von Histiozyten und Leukozyten gefressen werden und in den Lymphfollikeln und Bronchialepithelzellen auftreten und dass sie nach 3 Stunden nicht nur in den Alveolarlumen, und Bronchien von grossen mononukleären Zellen gefressen gefunden werden, sondern auch im Exsudat frei liegen.

4) Unter den Tuberkelbazillen im Alveolarlumen sind diejenigen, welche in den grossen mononukleären Zellen vorhanden sind, meist granulös; die im Exsudat frei liegenden aber kurzstabförmig, und zwar erscheinen sie im allgemeinen bis zum ungefähr 14 Tage nach der Injektion, wenn sie auch manchmal schon nach 3 Stunden erscheinen. Die Tuberkelbazillen in den Kapillaren, insbesondere in den kleinen Blutgefässen sind meist lang-oder kurzstabförmig und nehmen ungefähr 14 Tage nach der Injektion an schnell ab, wenn sie such nach. 7 Wochen noch spärlich vorhanden sind. Wenn man ferner die Tatsache berücksichtigt, dass die primären Zirkulationsstörungen, Hyperämie, Blutung, Ödem usw., überhaupt nur in dem obengenannten Stadium beobachtet werden, so kann man schliessen, dass die Tuberkelbazillen mit Ödemflüssigkeit direkt ins Alveolarlumen eindringen, ohne die Kapillaren zu zerstören. Da die Tuberkel im Alveolarlumen etwas später als die im Interstitium, d.h. 48 Stunden oder 13 Tage nach der Injektion auftritt, wird diese Reaktion wahrscheinlich, wie $\mathrm{T}$ a k e d a ${ }^{12}$ ) meinte, durch die ins Alveolarlumen gelangten Tuberkelbazillen verursacht. Die Tuberkelbazillen in den Bronchien sind wohl zum Teil aus dem Alveolarlumen ausgeschieden worden, aber zum Teil sind sie, nachdem einmal in die Bronchialepithelien gelangt, mit Abfall und Nekrose derselben im Bronchiallumen aufgetreten; denn die Bronchitis und der Abfall und die Nekrose der Brochchialepithelien werden bald nach der Bazilleninjektion wahrgenommen und dazu kommt noch dass, während die Tuberkelbazillen aus den Bronchien nachgewiesen werden, viele Bazillen in den Epithelzellen gefunden werden und später erheblich abnehmen. Dass die in dem Alveolarlumen und dem Bronchiallumen erschienenen Tuberkelbazillen teilweise durch die Luftwege in die Aussenwelt ausgeschieden werden, ist aus den Kulturergebnissen aus dem Bronchialsekret augenscheinlich. Dass die Tuberkelbazillen nach 4 Wochen und später noch aus den Bronchiallumen 
nachgewiesen werden und auch die Kultur positiv ausfällt, wird wahrscheinlich auf einer schon fortgeschrittenen tuberkulösen Veränderung. beruhen.

5. Schluss.

1) Führt man in die Ohrvene von Kaninchen hoch virulente Tuberkelbazillen vom Typus humanus ein, so treten die Hyperämie, das Ödem und teilweise Blutung auf. Gleichzeitig beginnt die Wucherung der fixen Gewebszellen und wird die Leukozyteninfiltration gewissermassen beobachtet.

2) Die pathologische Veränderung der Lunge, welche durch die in die Ohrvene eingespritzten Tuberkelbazillen hervorgerufen wird, ist hauptsächlich produktiv und die exsudstive Veränderung ist im allgemeinen die perifokale Pneumonie. Sie tritt im Interstitium des Alveolarseptums, insbesondere, in der Umgebung der Kapillaren, der Blutgefässe und Bronchien, und etwas später im Alveolarlumen auf.

3) Die Tuberkelbazillen im Blutstrom gelangen durch den Blutstrom in die Lunge, werden im Interstitium des Alveolarseptums und in den Kapillaren von den Leukozyten oder Histiozyten umgeben und gefressen oder erreichen die Lymphfollikel um die Bronchien, die Bronchialepithelien und etwas später mit Histiozyten oder Exsudat das Alveolarlumen. Durch den Abfall von den Bronchialepithelien treten sis zugleich auch in den Bronchiallumen auf. Das Stadium, in dem die Tuberkelbazillen im Alveolarlumen erscheinen, dauert gewöhnlich von 3 Stunde bis' zu 14 Tage nach der Injektion von Tuberkelbazillen. Nach 4 Wochen, wenn die Krankheit fortgeschritten ist, werden sie wieder in Bronchiallumen nachgewiesen.

4. Die ins Alveolarlumen eingedrungenen Tuberkelbazillen werden nur im Frühstadium, in dem verhältnismässig viele Bazillen im Blutstrom vorhanden sind, nachgewiesen.

An dieser Stelle möchte Verf. Herrn Prof. Dr. S. Nasu in dem pathologischen Institut für seine freundliche Anleitung und Anregung den aufrichtigsten Dank aussprechen.

Diese Forschung wurde durch Überlassung von Geldmitteln des Unterrichtsministeriums für wissenschaftliche Forschung ausgeführt, wofür herzlich gedankt sei.-Prof. Dr. T. Kumagai und 


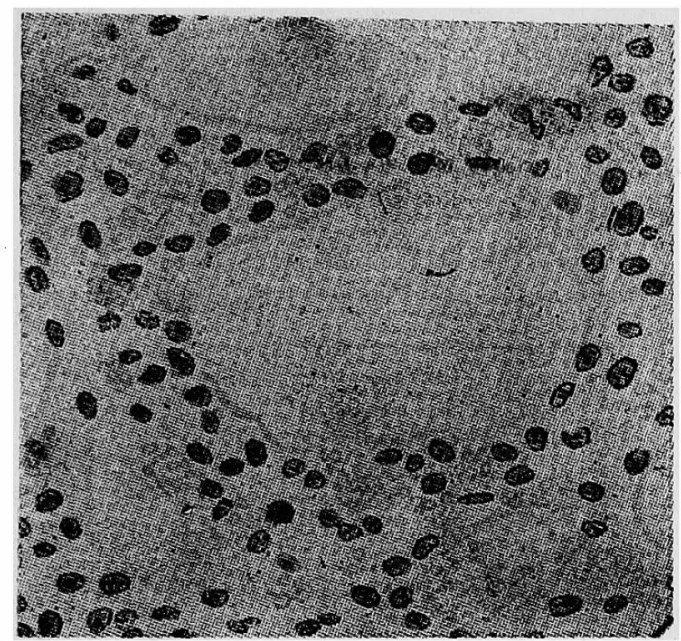

Fig. 1. Ein freier Tuberkelbazillus im Alveolarlumen der Lunge nach Ablauf von 72 Stunden bei der Injektion $1 \mathrm{mg}$ Bazillen. (1500:1)

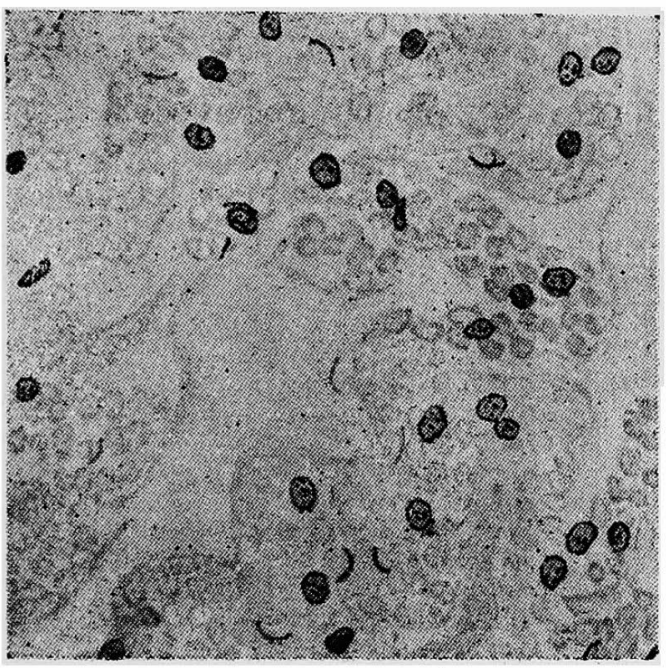

Fig. 2. Ein freier Tuberkelbazillus im Alveolarlumen der Lunge nach Ablauf von 3 Stunden bei der Injektion von $10 \mathrm{mg}$ Bazillen. (1500:1)

\section{Literatur.}

1) Baumgarte n, P., Verh. dtsch. path. Ges., 1902, 4, 2 u. 73.

2) Goyal, R.-K., C. r. Soc. Biol. Paris, 1936, 123, 379.

3) Wada, T., Igaku to Seibutugaku 1943, 4, 178.

4) Weigert, C., Verh. Ges. dtsch. Naturforsch., 1896, 668, 121.

5) Pagel, W., Beitr. klin. Tbk., 1925, 61, 220, 641, u. 678; 1926 63, 160.

6) Orth, J., Verh. dtsch. path. Ces., 1902, 4, 30.

7) Töppich, G., Krkh. forschg, 1926, 2, 15; 3, 335.

8) Taka mura, R., Nagasaki Igk. Z., 1938, 16, 373.

9) Miyata, S., Juzenkai Zassi, .1931, 36, 1239.

10) Huebschmann, P., Pathologische Anatomie der Tuberkulose, Berl., 1928, 191.

11) Takeda, K., Kekkaku, 1943, 21, 251. 